\title{
Caracterización fenotípica de aislamientos de Acinetobacter baumannii en una institución de salud de alta complejidad de Cali
}

\author{
Alfredo Pradoํ, Nhora L. Arias ${ }^{1}$, Mónica Chávez ${ }^{1,2}$, Cristina E. Cabrera' ${ }^{1,3}$, Romel F. Gómez ${ }^{1}$ \\ 1 Grupo de Investigación en Microbiología Molecular y Enfermedades Infecciosas, Facultad de Ciencias de la \\ Salud, Universidad Libre-Seccional Cali, Cali, Colombia \\ 2 Departamento de Ciencias Biomédicas, Facultad de Medicina, Universidad Santiago de Cali, Cali, Colombia \\ ${ }^{3}$ Departamento de Microbiología, Universidad del Valle, Cali, Colombia \\ El trabajo de laboratorio se realizó en el laboratorio del Grupo de Investigación en Microbiología Molecular y \\ Enfermedades Infecciosas de la Universidad Libre, Cali, Colombia.
}

Introducción. La caracterización fenotípica de las bacterias del género Acinetobacter mediante pruebas bioquímicas y microscópicas es posible. Varios estudios han demostrado que los aislamientos provenientes de infecciones asociadas a la atención en salud presentan una elevada resistencia a los antibióticos de primera elección.

Objetivo. Describir los patrones de resistencia de los aislamientos de Acinetobacter baumannii obtenidos en una institución de salud, así como sus características fenotípicas y los posibles mecanismos de resistencia.

Materiales y métodos. Se realizó un estudio descriptivo de corte transversal con 28 informes de muestras tomadas a pacientes hospitalizados con infección por $A$. baumannii. Las pruebas de sensibilidad para ceftazidime, cefepime, meropenem, amikacina y ciprofloxacina se realizaron con el sistema automatizado Vitek ${ }^{\circledR}$ y la clasificación de sensible, intermedia y resistente se hizo con base en el protocolo establecido por el Clinical and Laboratory Standards Institute para el año 2007.

Resultados. El mayor porcentaje de aislamientos correspondió al sexo masculino (53,6 \%), a la sala de infectología (28,5\%) y al mes de septiembre (21,4\%); el tipo de muestra más frecuente fue el de secreción endotraqueal (53,6\%). A partir de los patrones de los perfiles de sensibilidad a los antibióticos empleados se obtuvieron 13 filotipos.

Conclusión. Acinetobacter baumannii es un agente patógeno resistente a múltiples antimicrobianos, involucrado en brotes de infecciones asociadas a la atención en salud. Los patrones de los perfiles de resistencia permiten inferir que los posibles mecanismos de resistencia presentes en la mayoría de los aislamientos son la producción de betalactamasas de espectro extendido, las enzimas modificadoras del antibiótico y la modificación del sitio diana.

Palabras clave: Acinetobacter baumannii, farmacorresistencia microbiana, infección hospitalaria, pruebas de sensibilidad microbiana.

doi: http://dx.doi.org/10.7705/biomedica.v34i0.1666

Phenotypic characterization of Acinetobacter baumannii isolates in a high-complexity healthcare institution in the city of Cali

Introduction: Phenotypic characterization of the Acinetobacter genus bacteria through biochemical and microscopic tests is possible. Studies have shown that the isolates from health-care associated infections show high resistance to first-line antibiotics.

Objective: To describe the resistance patterns of the $A$. baumannii isolates obtained in a health care institution, the phenotypic characteristics of the isolates, and the possible resistance mechanisms.

Materials and methods: A descriptive cross-sectional study was conducted with 28 reports of samples taken from patients hospitalized with infection by $A$. baumannii. Susceptibility testing for ceftazidime, cefepime, meropenem, amikacin, and ciprofloxacin was performed with the Vitek $^{\mathrm{TM}}$ automated system and the susceptible, intermediate, and resistant classification was based on the protocol established by the Clinical and Laboratory Standards Institute for 2007.

Results: The highest percentage of isolates corresponded to males (53.6 \%), to the infectology ward

\section{Contribución de los autores:}

Romel Gómez: diseño del estudio.

Alfredo Prado, Mónica Chávez, Nhora Arias, Cristina E. Cabrera y Romel Gómez: análisis de los datos.

Todos los autores participaron en la elaboración del manuscrito. 
(28.5\%), and to the month of September (21.4\%); the most frequent sample site were endotracheal secretions (53.6\%). From the profile patterns for susceptibility to antibiotics used, 13 phylotypes were obtained.

Conclusion: Acinetobacter baumannii is a pathogen resistant to multiple antimicrobial agents involved in outbreaks of health-care associated infections. The resistance profile patterns allow inferring that the possible resistance mechanisms present in the majority of the isolates are: Production of extendedspectrum $\beta$-lactamases, antibiotic modifying enzymes, and target site modification.

Key words: Acinetobacter baumannii; drug resistance, microbial; cross infection, microbial sensitivity tests. doi: http://dx.doi.org/10.7705/biomedica.v34i0.1666

Las bacterias del género Acinetobacter son Gram negativas, aerobias estrictas, no fermentadoras, no móviles, positivas para catalasa y negativas para oxidasa, propiedades que permiten su caracterización fenotípica (1). Algunas especies de Acinetobacter tienen la capacidad de sobrevivir en condiciones extremas y por fuera de su huésped por periodos prolongados; se ha reportado la supervivencia de Acinetobacter iwoffi en superficies secas durante más de siete días, la de Acinetobacter calcoaceticus en superficies de fórmica hasta por 13 días y la de Acinetobacter baumannii en alimentos y elementos hospitalarios, entre otros, durante periodos mayores de 25 días (2-4); esto último hace posible la colonización de pacientes y personal de salud, lo que sumado a su capacidad para resistir la acción de la mayoría de los antibióticos de elección, incrementa la morbimortalidad hospitalaria (5).

En un estudio realizado en 139 hospitales de diferentes niveles de complejidad en países de Europa, Asia, Suráfrica, Australia, Norteamérica y Latinoamérica, Higgins, et al., reportaron en $A$. baumannii una resistencia de $96 \%$ a la combinación de betalactámicos con inhibidores de betalactamasas (piperacilina-tazobactam), a cefalosporinas de tercera generación (ceftazidime, $98 \%$ y ceftriazona, 99,6\%), así como resistencia a cefalosporinas de cuarta generación (cefepime, $96,1 \%$ ) y, finalmente, a aminoglucósidos (amikacina, $64,5 \%)(6)$.

Otros estudios realizados en Estados Unidos (7), Centroamérica (8) y Suramérica y los reportes de la Organización Panamericana de la Salud (OPS) para el año 2009 (9) dan cuenta del comportamiento de la resistencia en $A$. baummannii frente a los diferentes

\section{Correspondencia:}

Romel Fabián Gómez, Facultad de Ciencias de la Salud, Programa de Enfermería, Universidad Libre-Seccional Cali, Diagonal 37A № 3-29, Cali, Colombia

Teléfono: (572) 524 0007, extensión 1337

rofagom@gmail.com

Recibido: 17/05/13; aceptado: 12/11/13 grupos de antibióticos, registrándose los mayores porcentajes de resistencia a la combinación de betalactámicos con inhibidores de betalactamasas en Guatemala, $83 \%$, y Argentina, $85 \%$; en lo relacionado con la resistencia a cefalosporinas de tercera generación, cabe resaltar que en todo el continente se reportaron porcentajes por encima de $85 \%(7,9)$.

Los porcentajes de resistencia a cefalosporinas de cuarta generación en Estados Unidos y Centroamérica fueron de 85 y $89 \%$, respectivamente, mientras que en Argentina fue de $77 \%$. La resistencia a los carbapenem reportada en Estados Unidos fue de $50 \%$ (7), en tanto que para Centroamérica y Suramérica se ha reportado una resistencia por encima de $70 \%$ (9), similar al comportamiento de la resistencia a inhibidores de síntesis de proteínas (aminoglucósidos) y la resistencia a trimetropin-sulfametoxazol en Centroamérica y Suramérica (9).

En Bogotá se registraron resistencias de 91,6\% para aztreonam, $78 \%$ para piperacilina-tazobactam y $38,7 \%$ para imipenem (10). En Cali se reportaron porcentajes de resistencia de $82,5 \%$ para gentamicina, $77,8 \%$ para cefotaxima y $15,3 \%$ para imipenem en un estudio (11), mientras otro estudio reportó para este antibiótico una resistencia de 79,3\% (12).

Lo anterior evidencia la magnitud del problema de la resistencia de $A$. baumannii a los antibióticos en las instituciones prestadoras de servicios de salud de todo el mundo, incluida Colombia y la ciudad de Cali (6,9-12); esta situación reafirma la importancia que tiene la vigilancia epidemiológica de la resistencia a los antibióticos. En la literatura citada no se han señalado los perfiles de sensibilidad como una herramienta para la clasificación de los aislamientos en filotipos y la inferencia de los posibles mecanismos de resistencia que estos puedan expresar, lo cual consideramos como un posible aporte importante en la contención de la resistencia a los antibióticos de $A$. baumannii aislado en pacientes hospitalizados. 
El objetivo de este estudio fue describir los patrones de resistencia de los aislamientos de $A$. baumannii obtenidos en el año 2011, sus características fenotípicas y los posibles mecanismos de resistencia que pueden ser factores determinantes para las estrategias de control de infecciones.

\section{Materiales y métodos}

Se realizó un estudio descriptivo de corte transversal a partir de los informes del laboratorio clínico de muestras tomadas a pacientes hospitalizados entre enero y diciembre de 2011 con infección asociada a la atención en salud por $A$. baumannii en los diferentes servicios de una institución de salud de alta complejidad de Cali.

La institución presta servicios ambulatorios y hospitalarios, cuenta con tres unidades de cuidados intensivos, dos de cuidados especiales, seis quirófanos y 475 camas. Los usuarios pertenecen en su mayoría al régimen contributivo. La población atendida es en su mayoría adulta (84\%); se realizan en promedio 769 procedimientos quirúrgicos mensuales, incluidas cirugías de alta complejidad (13).

Se consideró infección asociada a la atención en salud aquella que se presentó en las 48 horas posteriores al ingreso del paciente a la institución hospitalaria (6); solo se incluyeron informes sobre aislamientos de $A$. baumannii. Se contemplaron variables relacionadas con el paciente, los servicios y las cepas. La fuente de información fue el informe del cultivo y el antibiograma del laboratorio de la institución, a partir del cual se generó una base de datos en Acces $2008^{\circledR}$ que incluyó las variables de sexo, sitio de muestra, servicio, período y patrón de sensibilidad.

Las pruebas de sensibilidad para los antibióticos ceftazidime, cefepime, meropenem, amikacina y ciprofloxacina se llevaron a cabo usando el sistema automatizado Vitek ${ }^{\circledR}$ y la clasificación de sensible (S), intermedia (I) y resistente (R) se hizo de acuerdo al protocolo establecido por el Clinical and Laboratory Standards Institute (CLSI) (14). Las cepas de Escherichia coli (ATCC 25922) y A. baumannii (ATCC 19606) se utilizaron como controles internos.

En el estudio se consideró filotipo a todos los aislamientos que presentaron el mismo perfil de sensibilidad a los antibióticos evaluados. Se asignó un número a cada grupo de aislamientos que presentara el mismo perfil de sensibilidad y con estos resultados se infirió el posible mecanismo de resistencia de cada filotipo.

\section{Resultados}

Se obtuvo información de 28 aislamientos provenientes de pacientes internados en los diferentes servicios; en el cuadro 1 se presentan las características de los pacientes y los sitios de toma de las muestras. El perfil de los pacientes correspondió a hombres $(53,6 \%, 15 / 28)$ hospitalizados con mayor frecuencia en los servicios de infectología $(28,5 \%, 8 / 28)$ y en unidad de cuidados intensivos (25\%,7/28); solo se reportó un aislamiento proveniente de un quirófano.

El número de muestras por mes osciló entre uno y seis, con un promedio de 2,8 y una mediana de muestras por mes de tres; en diciembre y julio no se reportaron datos sobre aislamientos microbiológicos de $A$. baumannii. El tipo de aislamiento más frecuente provenía de las secreciones $(53,6 \%, 15 / 28)$, seguido del hemocultivo $(17,8 \%$, $5 / 28$ ) mientras que en el caso de heridas, líquido peritoneal, punta de catéter y otro sitio sólo se reportó un aislamiento en cada uno.

Cuadro 1. Características de los pacientes y de los aislamientos clínicos de Acinetobacter baumannii, enero a diciembre de 2011, Cali, Colombia $(n=28)$

\begin{tabular}{|c|c|c|}
\hline Característica & $\mathbf{n}$ & $\%$ \\
\hline \multicolumn{3}{|l|}{ Sexo } \\
\hline Hombre & 15 & 53,6 \\
\hline Mujer & 13 & 46,4 \\
\hline \multicolumn{3}{|l|}{ Servicio } \\
\hline Infectología & 8 & 28,5 \\
\hline Unidad de cuidados intensivos & 7 & 25 \\
\hline Pediatría & 3 & 10,7 \\
\hline Medicina interna I & 6 & 21,4 \\
\hline Medicina interna II & 3 & 10,7 \\
\hline Quirófanos & 1 & 3,5 \\
\hline \multicolumn{3}{|l|}{ Mes } \\
\hline Septiembre & 6 & 21,4 \\
\hline Mayo & 4 & 14,3 \\
\hline Febrero & 3 & 10,7 \\
\hline Junio & 3 & 10,7 \\
\hline Agosto & 3 & 10,7 \\
\hline Noviembre & 3 & 10,7 \\
\hline Enero & 2 & 7,1 \\
\hline Octubre & 2 & 7,1 \\
\hline Marzo & 1 & 3,5 \\
\hline Abril & 1 & 3,5 \\
\hline \multicolumn{3}{|l|}{ Sitio de muestra } \\
\hline Secreción endotraqueal & 15 & 53,6 \\
\hline Hemocultivo & 5 & 17,8 \\
\hline Orina, micción espontánea & 2 & 7,1 \\
\hline Orina de sonda & 2 & 7,1 \\
\hline Herida & 1 & 3,5 \\
\hline Líquido peritoneal & 1 & 3,5 \\
\hline Punta de catéter & 1 & 3,5 \\
\hline Otro tipo & 1 & 3,5 \\
\hline
\end{tabular}

Fuente: informes microbiológicos, IPS de alta complejidad, 2011 
En el cuadro 2 se presentan los registros de sensibilidad y resistencia para cada antibiótico; la mayor resistencia se presentó frente a cefepime $(82,1 \%, 23 / 28)$, mientras que el ceftazidime solo se notificó como resistente en 14,3 \% (4/28) de los reportes. Por el contrario, la mayor sensibilidad se dio frente a ceftazidime (50\%, 14/28), mientras que solo un aislamiento resultó sensible a la ciprofloxacina.

En el cuadro 3 se describen los 13 filotipos obtenidos, siendo el número 3 y el número 4 los más frecuentes, pues se encontraron en nueve y seis aislamientos, respectivamente. El filotipo 3 tenía un patrón de resistencia a las cefalosporinas de cuarta generación, los carbapenem, los aminoglucósidos y las quinolonas, con sensibilidad solamente frente a ceftazidime, cefalosporina de tercera generación, mientras que el filotipo 4 mostró resistencia a cefalosporinas de cuarta generación, quinolonas y aminoglucósidos y sensibilidad intermedia a cefalosporinas de tercera generación como el

Cuadro 2. Patrón de sensibilidad a los antibióticos, enero a diciembre de 2011, Cali, Colombia $(n=28)$

\begin{tabular}{lrrrr}
\hline $\begin{array}{l}\text { Antibiótico/ } \\
\text { patrón }\end{array}$ & Resistente & Sensible & Intermedio & ND \\
\hline CAZ & 4 & 14 & 10 & 0 \\
FEP & 23 & 4 & 1 & 0 \\
MEM & 20 & 4 & 0 & 4 \\
AMK & 22 & 5 & 1 & 0 \\
CIP & 21 & 1 & 0 & 0 \\
\hline
\end{tabular}

AMK: amikacina; FEP: cefepime; CAZ: ceftazidime; MEM: meropenem; CIP: ciprofloxacina; ND: ningún dato

Fuente: informes microbiológicos, IPS de alta complejidad, 2011

Cuadro 3. Filotipos de aislamientos clínicos de A. baumanni, enero a diciembre de 2011, Cali, Colombia $(n=28)$

\begin{tabular}{rcccccc}
\hline Filotipo & $\begin{array}{c}\text { Número de } \\
\text { informes }\end{array}$ & CAZ & FEP & MEM & AMK & CIP \\
\hline 1 & 2 & $\mathrm{R}$ & $\mathrm{R}$ & $\mathrm{R}$ & $\mathrm{R}$ & $\mathrm{R}$ \\
2 & 1 & $\mathrm{R}$ & $\mathrm{R}$ & $\mathrm{R}$ & $\mathrm{S}$ & $\mathrm{R}$ \\
3 & 9 & $\mathrm{~S}$ & $\mathrm{R}$ & $\mathrm{R}$ & $\mathrm{R}$ & $\mathrm{R}$ \\
4 & 6 & $\mathrm{I}$ & $\mathrm{R}$ & $\mathrm{R}$ & $\mathrm{R}$ & $\mathrm{R}$ \\
5 & 1 & $\mathrm{~S}$ & $\mathrm{I}$ & $\mathrm{R}$ & $\mathrm{R}$ & $\mathrm{R}$ \\
6 & 1 & $\mathrm{I}$ & $\mathrm{R}$ & $\mathrm{R}$ & $\mathrm{I}$ & $\mathrm{R}$ \\
7 & 1 & $\mathrm{I}$ & $\mathrm{R}$ & $\mathrm{S}$ & $\mathrm{S}$ & $\mathrm{R}$ \\
8 & 1 & $\mathrm{I}$ & $\mathrm{R}$ & $\mathrm{ND}$ & $\mathrm{R}$ & $\mathrm{R}$ \\
9 & 1 & $\mathrm{R}$ & $\mathrm{R}$ & $\mathrm{ND}$ & $\mathrm{R}$ & $\mathrm{R}$ \\
10 & 1 & $\mathrm{~S}$ & $\mathrm{~S}$ & $\mathrm{ND}$ & $\mathrm{R}$ & $\mathrm{R}$ \\
11 & 1 & $\mathrm{I}$ & $\mathrm{R}$ & $\mathrm{ND}$ & $\mathrm{R}$ & $\mathrm{R}$ \\
12 & 2 & $\mathrm{~S}$ & $\mathrm{~S}$ & $\mathrm{~S}$ & $\mathrm{~S}$ & $\mathrm{R}$ \\
13 & 1 & $\mathrm{~S}$ & $\mathrm{~S}$ & $\mathrm{~S}$ & $\mathrm{~S}$ & $\mathrm{~S}$ \\
\hline
\end{tabular}

AMK: amikacina; FEP: cefepime; CAZ: ceftazidime; MEM: meropenem; CIP: ciprofloxacina; ND: ningún dato

Fuente: informes microbiológicos, IPS de alta complejidad, 2011 ceftazidime. Los filotipos 2 y 5 y del 6 al 11 presentaron resistencia a amikacina o a alguno de los betalactámicos. El filotipo 1 mostró resistencia a todos los antibióticos; por otro lado, el filotipo 13 mostró un patrón de sensibilidad a todos los antibióticos (cuadro 3).

\section{Discusión}

En este estudio el tipo de aislamiento más frecuente se obtuvo de secreciones endotraqueales, situación que puede estar relacionada con los prolongados periodos de respiración mecánica asistida a que deben someterse los pacientes en las unidades de cuidados intensivos (15).

Al menos, 68 \% (19/28) de los aislamientos presentó resistencia a cuatro antibióticos o más de los usados como primera elección en el tratamiento de infecciones con $A$. baumannii; este resultado es superior a lo reportado en Europa $(16,17)$, Estados Unidos (7), Centroamérica (8), Suramérica (18) y en el resto del Valle del Cauca $(11,12)$, pero está por debajo de lo reportado en Bogotá (10).

El bajo porcentaje de aislamientos resistentes a ceftazidime (14\%, 4/28) agrupados en los filotipos 1 , 2 y 9 contrasta con lo encontrado en Europa $(16,17)$, Estados Unidos $(6,7)$, Centroamérica $(6,8,9)$, otros países de Suramérica $(9,18)$ e incluso en otros estudios realizados en Colombia, en los cuales el porcentaje de resistencia a este antibiótico ha estado siempre por encima de $60 \%$ (10).

El $86 \%(24 / 28)$ de los aislamientos (filotipos 1 al 9 y 11) mostró resistencia o sensibilidad intermedia al cefepime, lo que coincide con lo encontrado en Europa $(16,17)$, Estados Unidos $(6,7)$ y Centroamérica $(8,9)$, pero está por encima de lo informado en otros países de Suramérica (9) y en otros estudios realizados en Colombia (10-12).

A pesar de que cuatro de los aislamientos no presentaron valores de sensibilidad frente a los carbapenem, $72 \%$ (filotipos 1 al 6) resultó resistente a meropenem, cifra superior a lo reportado en los últimos tres años en Europa $(16,17)$, Estados Unidos $(6,7)$ y otros países de Suramérica (9), incluido Colombia (9-12), y similar a lo reportado en países de Centroamérica para el 2009 (9), lo que evidencia un incremento en la resistencia a este antibiótico (19).

El $79 \%(22 / 28)$ de los aislamientos (filotipos 1, 3 a 5 y 9 a 11) mostró resistencia a amikacina, cifra superior a la reportada por los Estados Unidos $(6,7)$, pero similar a la de países de Centroamérica 
$(8,9)$ y Suramérica $(9)$, y menor a los resultados de estudios realizados en Colombia (10), incluso a lo reportado en el 2010 en Cali (12).

El 96 \% (27/28) de los aislamientos mostró resistencia a ciprofloxacina; es decir, solo un aislamiento (filotipo) se mostró sensible a este antibiótico. Este resultado es mayor que el reportado por países de Centroamérica (8), pero similar a lo reportado por algunos países de Suramérica (9), y superior a lo reportado en Colombia (10), aunque similar a lo informado en Cali para el 2010 (12).

La resistencia a ceftazidime observada en los filotipos 1, 2 y 9 podría deberse a la producción excesiva de AmpC o bien a la presencia de una enzima betalactamasa de espectro extendido de tipo OXA de clase molecular D (20-22). Por otro lado, la resistencia de los filotipos 1 y 9 a los aminoglucósidos (amikacina) podría responder a la producción de una enzima modificadora codificada en plásmidos (aminoglucósido3-fosfotransferasa IV)(23,24) y la resistencia a las quinolonas (ciprofloxacina) podría estar relacionada con una mutación en el gen gyrA, el cual fue reportado en $A$. baumannii por Vila, et al., en un estudio realizado en España en 1995 $(25,26)$.

Algunos autores mencionan que entre los aislamientos clínicos de $A$. baumannii pueden aparecer algunos que sintetizan carbapenemasas de clase B, tipo IMP, los cuales muestran una actividad marcada frente a los diferentes grupos de betalactámicos; este podría ser el caso de los filotipos 1,2 y $9(27,28)$, los cuales se mostraron resistentes a cefalosporinas de tercera y cuarta generación. En cuanto a la resistencia a los carbapenem, el mecanismo más prevalente en esta especie es la producción de OXA carbapenemasas y la combinación de mecanismos (5).

El $84 \%$ de los aislamientos (filotipos 1 al 4, 6 al 9 y 11) mostró resistencia a las cefalosporinas de cuarta generación, específicamente a cefepime; por ser este un betalactámico, es probable que la resistencia a este pero no a ceftazidime se deba a la presencia de un tipo de betalactamasa de espectro extendido denominada RTG-4 o CARB10 (29). Además, los estudios realizados por Doi, et al., demostraron la presencia de las variantes de la betalactamasa CMY-2 (CMY-33 Y CMY44) que confieren resistencia al cefepime (30), lo que explicaría la resistencia observada en los aislamientos de los filotipos aquí mencionados.
El filotipo 7 mostró resistencia al cefepime, lo que puede deberse a la presencia de una betalactamasa de tipo AmpC de espectro extendido (31). Por otro lado, a pesar de que las betalactamasas de tipo AmpC presentan baja afinidad por los carbapenem, los mecanismos conjuntos como la hiperproducción de la enzima y el cierre de porinas por parte de la bacteria, así como la baja cantidad del antibiótico a nivel periplásmico, permiten que la enzima hidrolice el antibiótico y se registre resistencia a los carbapenem, tal como se observó en este filotipo $(32,33)$. Igualmente, Fernández, et al., afirman que la ausencia de dos proteínas unidoras de penicilinas, una de 73,2 kDa (PUP2a) y otra de $70,1 \mathrm{kDa}$ (PUP2b), se relaciona con la resistencia a imipenem, meropenem o a ambos, lo que podría explicar la resistencia de este filotipo a los carbapenem, específicamente a meropenem (34).

Una de las limitaciones de este estudio se relaciona con el uso de fuentes secundarias de información que no permitieron obtener más datos sobre las características de los pacientes ni sobre los perfiles de sensibilidad, específicamente a meropenem. Por lo tanto, no fue posible obtener información más exacta sobre este antibiótico que, según la Red de Eventos Nosocomiales del Valle del Cauca, registró para este mismo año un porcentaje de resistencia en $A$. baumanii de $74 \%$ (35).

A partir de los hallazgos de este estudio se propone la realización de pruebas moleculares a fin de establecer la relación entre los perfiles de sensibilidad y los posibles mecanismos de resistencia. Además, sería importante indagar sobre la relación entre la presencia de resistencia y el manejo de dispositivos, procedimientos y demás actividades realizadas por el personal de salud involucrado en la atención de los pacientes en los sitios de infección, así como las medidas preventivas que pueden tomarse para evitar la diseminación de este tipo de microorganismos.

En conclusión, los aislamientos del filotipo 1 resistentes a todos los antibióticos podrían presentar diversos mecanismos de resistencia a antibióticos. Podría afirmarse que los patrones de los perfiles de resistencia permiten inferir los posibles mecanismos de resistencia presentes en la mayoría de los aislamientos.

Los elevados porcentajes de resistencia a los antibióticos probados sugeriría que el personal de salud se está quedando sin opciones terapéuticas para ofrecer una adecuada intervención a los pacientes con infecciones asociadas a la atención en salud causadas por $A$. baumannii. 


\section{Agradecimientos}

A la Corporación Comfenalco Valle, Universidad Libre (Clínica Universitaria Rafael Uribe Uribe).

\section{Conflicto de intereses}

Los autores declaran no tener conflicto de intereses con la institución en donde fueron obtenidos los datos.

\section{Financiación}

Esta investigación fue financiada en su totalidad por la Universidad Libre, seccional Cali, concretamente por la Facultad de Ciencias de la Salud, Programa de Enfermería, a través del Plan Integral de Desarrollo Institucional (PIDI).

\section{Referencias}

1. Peleg A, Seifert H, Paterson D. Acinetobacter baumannii emergence of a successful pathogen. Clin Microbiol Rev. 2008;2:538-82. http://dx.doi/10.1128/CMR.00058-07

2. Diomedi A. Infecciones por Acinetobacter baumannii panresistente. Consideraciones epidemiologicas y de manejo antimicrobiano actualizado. Rev Chil Infectol. 2005;22:298320. http://dx.doi.org/10.4067/S0716-10182005000600003

3. Berlau BJ, Aucken HM, Houang E, Pitt TL. Isolation of Acinetobacter spp. Including $A$. baumannii from vegetables implications for hospital-acquired infections. J Hosp Infect. 1999;42:201-4. http://dx.doi.org/10.1053/jhin.1999.0602

4. Giamarellou H, Antoniadou A, Kanellakopoulou K. Acinetobacter baumannii a universal threat to public health. Int J Antimicrob Agents. 2008;32:106-19. http://dx.doi.org/ 10.1016/j.ijantimicag.2008.02.013

5. Villegas MV, Kattan J, Correa A, Lolans K, Guzmán AM, Woodford N. Dissemination of Acinetobacter baumannii clones with OXA-23 carbapenemase in Colombian hospitals. Antimicrob Agents Chemother. 2007;51:2001-4. http://dx. doi.org/10.1128/AAC.00226-07

6. Higgins PG, Dammhayn C, Hackel M, Seifert H. Global spread of carbapenem resistant Acinetobacter baumannii. J Antimicrob Chemother. 2010;65:233-8. http://dx.doi.org/ 10.1093/jac/dkp428

7. Valentine SC, Contreras D, Tan S, Real LJ, Chu S, Howard X. Phenotypic and molecular characterization of Acinetobacter baumannii clinical isolates from nosocomial outbreaks in Los Angeles County California. J Clin Microbiol. 2008;46:2499-507. http://dx.doi.org/10.1128/JCM.00367-08

8. Hart M, Llanes N, Espinosa F, Halley MC, Martínez ML, López AL. Estudio de la sensibilidad antimicrobiana de la especie Acinetobacter baumanii en el Hospital "Hermanos Ameijeiras", año 2006. Rev Cubana Med. 2008;47:1-9.

9. Organización Panamericana de Salud. Informe anual de la Red de Monitoreo de Vigilancia de la Resistencia a los Antibióticos. OPS/HDM/CD/A/541/09 2009. Washington, D.C.: Organización Panamericana de la Salud; 2011.

10. Pinzón JO, Mantilla JR, Valenzuela EM, Fernández F, Álvarez CA, Osorio EJ. Caracterización molecular de aislamientos de Acinetobacter baumannii provenientes de la unidad de quemados de un hospital de tercer nivel de Bogotá. Infectio. 2006;10:71-8.

11. Miranda MC. Estudio de la epidemiología molecular y de los factores de riesgo asociados a aislamiento de Acinetobacter baumannii en pacientes hospitalizados en el Hospital Universitario del Valle (tesis). Cali: Universidad del Valle; 2005.

12. Chávez M, Salazar M, Cabrera C, Gómez R, Pallares C. Bacterias resistentes a los antibióticos en infecciones nosocomiales de un hospital en Colombia. Enf Inf Microbiol. 2012;33:19-25.

13. Matta L, Martínez DM, Rendón LF, León H. Comportamiento de las infecciones nosocomiales en la Corporación Comfenalco Valle - Universidad Libre (julio de 2009 - junio de 2010). Rev Col Salud Libre. 2010;8:100-7.

14. Clinical and Laboratory Standards Institute. Performance standards for antimicrobial susceptibility testing. Seventeenth Informational Supplement. Wayne, PA: CLSI; 2007.

15. Montero JC, Ortíz E, Fernández T, Cayuela A, Marque J, García A, et al. Acinetobacter baumannii ventilatorassociated pneumonia: Epidemiological and clinical findings. Intensive Care Med. 2005;31:649-55. http://dx.doi.org/10. 1007/s00134-005-2598-0

16. Gallegoa L, Canduelaa MJ, Sevillanoa E, Pujanaa I, Calvobf F, Adelai F. Detección de carbapenemasas en clones de Acinetobacter baumannii resistentes a imipenem. Enferm Infec Microbiol Clin. 2004;22:262-6.

17. Picazo JJ, Betriu C, Rodríguez-Avial I, Culebras E, Gómez M, López F. Antimicrobial resistance surveillance: VIRA STUDY 2006. Enferm Infecc Microbiol Clin. 2006;24:617-28.

18. von Dolinger de Brito $\mathrm{D}$, Oliveira EJ, Abdallah VO, da Costa Darini AL, Gontijo Filho PP. An outbreak of Acinetobacter baumannii septicemia in a neonatal intensive care unit of a university hospital in Brazil. Braz $\mathrm{J}$ Infect Dis. 2005;9:301-9. http://dx.doi.org/10.1590/S141386702005000400006

19. Livermore DM. The impact of carbapenemases on antimicrobial development and therapy. Curr Opin investig Drugs. 2002;3:218-24.

20. Navia MM, Ruiz J, Vila J. Characterization of an integron carrying a new class $D$ betalactamase (OXA-37) in Acinetobacter baumannii. Microb Drug Resist. 2002;8:261-5. http://dx.doi.org/10.1089/10766290260469516

21. Bou G, Martínez-Beltrán J. Cloning nucleotide sequencing and analysis of the gene encoding an AmpC $\beta$-lactamase in Acinetobacter baumannii. Antimicrob Agents Chemother. 2000;44:428-32. http://dx.doi.org/10.1128/AAC.44.2.428-432. 2000

22. Danes C, Navia MM, Ruiz J, Marco F, Jurado A, Jiménez de Anta MT. Distribution of $\beta$-lactamases in Acinetobacter baumannii clinical isolates band the effect of Syn 2190 (AmpC inhibitor) in the mics of different beta-lactam antibiotics. J Antimicrob Chemother. 2002;50:261-4. http://dx. doi.org/10.1093/jac/dkf092

23. Vila J, Ruiz J, Navia M, Becerril B, García I, Perea S. Spread of amikacin resistance in Acinetobacter baumannii strains isolated in Spain due to an epidemic strain. J Clin Microbiol. 1999;37:758-61.

24. Vila J. Mechanisms of antimicrobial resistance in Acinetobacter baumannii. Rev Med Microbiol.1998; 9:87-97. 
25. Vila J, Ruiz J, Goñi $\mathbf{P}$, Jiménez de Anta MT. Mutation in the gyrA gene of quinolone resistant clinical isolates of Acinetobacter baumannii. Antimicrob Agents Chemother. 1995;39:1201-3.

26. Vila J , Ruiz J , Goñi P, Jiménez de Anta T. Quinoloneresistance mutations in the topoisomerase IV parC gene of Acinetobacter baumannii. J Antimicrob Chemother. 1997; 39:757-62. http://dx.doi.org/10.1093/jac/39.6.757

27. Riccio ML, Franceschini N, Boschi L, Carravelli B, Cornaglia G, Fontana R, et al. Characterization of the metallo- $\beta$-lactamase determinant of Acinetobacter baumannii a C-5 4 / 97 reveals the existence of blalMP allelic variants carried by gene cassettes of different phylogeny. Antimicrob Agents Chemother. 2000;44:1229-35.

28. Chu YW, Afzal-Shah M, Houang ET, Palepou MF, Lyon DJ, Woodford N, et al. IMP-4, a novel metallo- $\beta$-lactamase from nosocomial Acinetobacter spp collected in Hong Kong between 1994 and 1998. Antimicrob Agents Chemother. 2001;45:710-4. http://dx.doi.org/10.1128/AAC.45.3.710-714.2001

29. Nordmann P, Poirel L. Acinetobacter baumannii basic and emerging mechanisms of resistance. Eur Infect Dis. 2008;2:94-7.

30. Doi Y, Paterson DL. Detection of plasmid-mediated class $C$ B-lactamases. Int J Infect Dis. 2007;11:191-7. http://dx.doi. org/10.1016/j.ijid.2006.07.008
31. Martínez DV. Betalactamasas tipo AmpC. Generalidades y métodos para detección fenotípica. Rev Soc Ven Microbiol. 2009;29:1315-2556.

32. Lee EH, Nicolas MH, Kitzis MD, Pialoux G, Collatz E, Gutmann L. Association of two resistance mechanisms in a clinical isolate of Enterobacter cloacae with high-level resistance to imipenem. Antimicrob Agents Chemother. 1991;35:1093-8. http://dx.doi.org/10.1128/AAC.35.6.1093

33. Bradford PA, Urban C, Mariano N, Projan SJ, Rahal JJ, Bush K. Imipenem resistance in Klebsiella pneumoniae is associated with the combination of ACT-1, a plasmid mediated AmpC betalactamase, and the loss of an outer membrane protein. Antimicrob Agents Chemother. 1997;41:563-9.

34. Fernández F, Martínez L, Conejo MC, Ayala JA, Perea EJ, Pascual A. Relationship between betalactamase production, outer membrane protein and penicillin binding protein profiles on the activity of carbapenems against clinical isolates of Acinetobacter baumannii. J Antimicrob Chemother. 2003;51:565-74. http://dx.doi.org/10.1093/jac/ dkg097

35. Red de Vigilancia de Eventos Nosocomiales del Valle. Perfil Epidemiológico en 15 Instituciones IPS nivel 3 de la Ciudad de Cali, periodo enero-diciembre 2011. Fecha de consulta: 12 de febrero de 2013. Disponible en: http:// www.renova.org.co/sitio/images/Presentaciones/Informe_ RENOVA_2011_V5.pdf. 